\title{
Stability Analysis for Fruit Yield and its Components in Tomato (Solanum lycopersicum L.) under Acidic Soils of Manipur Valley
}

\author{
N. Brajendra Singh*, Joyashree Dey, Herojit Singh Athokpam and J. M. Laishram \\ College of Agriculture, CAU, Imphal, Manipur, India \\ *Corresponding author
}

\section{A B S T R A C T}

\begin{tabular}{l} 
K e y w o r d s \\
$\begin{array}{l}\text { Stability, Solanum } \\
\text { lycopersicum L., } \\
\text { Acidic soils, } \\
\text { Manipur valley }\end{array}$ \\
\hline Article Info \\
$\begin{array}{l}\text { Accepted: } \\
\text { 15 December } 2020 \\
\text { Available Online: } \\
\text { 10 January } 2021\end{array}$ \\
\hline
\end{tabular}

\section{Introduction}

High yielding varieties that have inherent stability in performance over different agroecological conditions are of great significance in countries like India towards sustainable production and productivity. The phenotypic performance of a genotype is not necessarily the same under diverse agro-ecological conditions. Some genotypes may perform well in some environments but not so well in others. Such genotype and environment (Gx E) interactions have assumed greater importance in Plant Breeding as they affect stability of genotypes under diverse environments. Once the $\mathrm{G} \times \mathrm{E}$ interactions were found to be significant, the next task is to identify stable genotypes. The stable genotypes are one which interact less with the environments giving a near consistent performance across different environments (Finlay and Wilkinson, 1963). A variety is said to be stable when regression coefficient (b) is equal to one and deviation from regression $\left(\mathrm{S}^{2} \mathrm{~d}\right)$ as close to zero as possible with high mean performance (Eberhart and Russell, 1966). Allard and Bradshaw (1964) suggested selection of stable genotypes that interact less with environments in which they are to be grown with a view to reduce the genotype $\mathrm{x}$ environment interaction to a considerable extent. The above three 
measures of assessing the stability of genotype viz, mean, regression coefficient (b) and the mean square deviation $\left(\mathrm{S}^{2} \mathrm{~d}\right)$ were employed in assessing the stability of genotypes included in the present study.

In the present investigation, an attempt has been made to study the stability of some promising genotypes of tomato (Solanum lycopersicum L.) under acidic soils of Manipur.

\section{Materials and Methods}

The materials of the present investigation comprised 29 genotypes of tomato possessing different morphological and productive attributes. The genotypes were studied for stability parameters under five different environments viz., September 2017 (E1), November 2017 (E2), February 2018 (E3), September 2018 (E4) and November 2018 (E5). The experiment was laid out in Randomised Block Design with three replications at experimental farm of College of Agriculture, CAU, Imphal. The 25 days old seedlings were transplanted in 6 rows of $2 \mathrm{~m}$ length maintaining plant to plant and row to row distances of $40 \mathrm{~cm}$ and $60 \mathrm{~cm}$ respectively in each replication. All the recommended package of practices was followed to raise the crop. Observations were recorded on 10 randomly chosen plants in each replication for plant height, number of primary branches per plant, number of fruits per plant, length of the fruit, fruit diameter, fruit weight, fruit yield per plant, while for days to $50 \%$ flowering and days to maturity, data were recorded on plot basis. Stability parameters were estimated using the model proposed by Eberhart and Russel (1966).

\section{Results and Discussion}

Pooled analysis of variance revealed significant differences among the genotypes for most of the characters under study. Further, significant variances due to environment (linear) indicated that performance of the genotypes could be predicted. The variance due to $\mathrm{G} \times \mathrm{E}$ was significant for all the characters except numbers of primary branches per plant, fruit length; fruit weight and fruit yield per plant (Table 1). Stability parameters for different characters are summarised in table 2 .

Most stable genotype among all the studied genotypes for days to $50 \%$ flowering was TWC-3 with unit regression coefficient and non- significant deviation from regression.

For days to maturity the genotype Pusa Ruby was the only genotype, which was stable having mean value lower than the population mean with unit regression and non-significant deviation from regression.

Among the genotypes studied for plant height at maturity, most stable genotypes were CAUM-4, CAU-TS-2, CAU-TS-4 and TWC-2. These genotypes expressed lower plant height from the population mean with unit regression coefficient and non- significant deviations from the regression.

For the character number of primary branches per plant mean sum of squares for [Environment + Genotype $\mathrm{x}$ environment] as well as Environment (linear) and Genotype $\mathrm{x}$ Environment (linear) were non-significant. This clearly showed that the genotypes did not respond significantly to varying degrees of environment i.e. over different sowing dates and year. Among the genotypes, most stable were Pusa Ruby and Money Maker with unit regression coefficient and nonsignificant deviation from regression. In the study of the most important yield contributing character viz., number of fruits per plant, the most stable genotypes were BRH-2, Sutton Roma, Marglobe Supreme and CAU-TS-5. 
Table.1 Pooled analysis of variance over all environments for different characters in tomato (Eberhart and Russell, 1966)

\begin{tabular}{|c|c|c|c|c|c|c|c|c|c|c|}
\hline \multirow[b]{2}{*}{$\begin{array}{l}\text { Source of } \\
\text { variation }\end{array}$} & \multirow[b]{2}{*}{ d.f } & \multicolumn{9}{|c|}{ Mean sum of squares } \\
\hline & & $\begin{array}{l}\text { Days to } \\
50 \% \\
\text { flowering }\end{array}$ & $\begin{array}{l}\text { Days to } \\
\text { maturity }\end{array}$ & $\begin{array}{l}\text { Plant } \\
\text { height }\end{array}$ & $\begin{array}{c}\text { Number } \\
\text { of primary } \\
\text { branches per } \\
\text { plant }\end{array}$ & $\begin{array}{l}\text { Number of } \\
\text { fruits per } \\
\text { plant }\end{array}$ & Fruit length & $\begin{array}{c}\text { Fruit } \\
\text { diameter }\end{array}$ & $\begin{array}{c}\text { Fruit } \\
\text { weight }\end{array}$ & $\begin{array}{c}\text { Fruit yield } \\
\text { per plant }\end{array}$ \\
\hline Genotype (G) & 28 & $303.12 * *$ & $454.62 * *$ & 38.53 & 0.73 & $51.97 * *$ & $1.12 *$ & $0.93 * *$ & $303.14 * *$ & $31650.30 * *$ \\
\hline $\begin{array}{l}\text { Environment } \\
\text { (E)+ }\end{array}$ & 116 & 16.90 & 20.98 & 54.15 & 0.13 & 2.67 & 0.24 & 0.22 & 41.24 & 2807.44 \\
\hline$(\mathbf{G} \times \mathbf{E})$ & 1 & 17.84 & 75.40 & $594.24 * *$ & 1.07 & $26.79 * *$ & $1.39 * *$ & $3.28 * *$ & 16.34 & 8654.60 \\
\hline E (Linear) & 28 & 48.83* & $68.57 * *$ & $173.45 * *$ & 0.14 & $8.83 *$ & 0.52 & $0.76 * *$ & 35.26 & 4129.13 \\
\hline G X E (Linear) & 87 & $84.30 * *$ & $58.95 * *$ & $163.03 * *$ & $1.31 * *$ & 7.14 & $1.49 * *$ & 0.50 & $436.24 * *$ & 2413.35 \\
\hline $\begin{array}{l}\text { Pooled deviation } \\
\text { Pooled error }\end{array}$ & 280 & 28.78 & 37.29 & 76.23 & 0.60 & 5.61 & 0.45 & 0.34 & 86.97 & 9774.95 \\
\hline
\end{tabular}

$*$,** Significant at $\mathrm{P}=0.05$ and $\mathrm{P}=0.01$ level respectively

Table.2 Stability parameters for different characters in tomato

\begin{tabular}{|l|c|c|c|c|c|c|c|c|c|}
\hline & \multicolumn{3}{|c}{ Days to 50\% flowering } & \multicolumn{3}{c|}{ Days to maturity } & \multicolumn{3}{c|}{ Plant height (cm) } \\
\hline Genotype & Mean & bi & $\mathrm{S}^{2} \mathrm{di}$ & Mean & bi & $\mathrm{S}^{2} \mathrm{di}$ & Mean & bi & $\mathrm{S}^{2} \mathrm{di}$ \\
\hline TWC-1 & 71.27 & 0.4857 & -9.07 & 38.73 & 3.195 & -12.51 & 71.47 & 3.5060 & -21.01 \\
\hline TWC-2 & 64.95 & 1.3273 & -6.92 & 95.87 & 0.491 & -9.78 & 70.22 & -2.6850 & -11.45 \\
\hline TWC-3 & 61.44 & 0.807 & 1.16 & 91.93 & 3.698 & -12.03 & 65.32 & 0.2860 & -24.53 \\
\hline TWC-4 & 68.32 & -1.1267 & 5.3 & 94.20 & -5.788 & -0.16 & 61.97 & -1.5520 & -19.46 \\
\hline TWC-8 & 77.67 & -1.1059 & -0.37 & 99.33 & 0.428 & -8.37 & 60.79 & 0.9930 & -21.2 \\
\hline TWC-9 & 84.17 & -0.1134 & -3.68 & 97.73 & 1.102 & -8.34 & 63.96 & -0.5830 & -1.62 \\
\hline TWC-10 & 82.26 & 0.4516 & -0.94 & 97.33 & 0.324 & -2.37 & 74.46 & 4.7150 & -23.84 \\
\hline TWC-11 & 76.75 & 1.2078 & -8.5 & 90.87 & 4.732 & -4.09 & 72.26 & 4.5690 & -52.09 \\
\hline A.ALOK & 69.99 & -0.1151 & 21.48 & 89.80 & 0.004 & -0.09 & 75.32 & 4.4610 & -20.87 \\
\hline A.VIKAS & 81.75 & -2.5612 & 63.73 & 106.00 & -19.196 & 16.51 & 71.45 & -4.3330 & 34.93 \\
\hline BRH-1 & 92.26 & 0.2047 & -8.93 & 120.67 & 1.095 & -9.9 & 56.93 & -1.1150 & -13.13 \\
\hline
\end{tabular}




\begin{tabular}{|l|c|c|c|c|c|c|c|c|c|}
\hline BRH-2 & 90.20 & 0.65 & -9.01 & 117.53 & 3.318 & 18.22 & 52.86 & -0.0999 & -7.26 \\
\hline PED & 84.33 & 0.7921 & -0.93 & 105.73 & 7.723 & -6.02 & 66.61 & 5.3120 & 8.03 \\
\hline PR & 74.05 & 1.5931 & 24.34 & 98.00 & 2.005 & -11.84 & 72.94 & -2.2470 & -22.79 \\
\hline SR & 68.29 & 0.2849 & -0.24 & 96.60 & 1.019 & -11.74 & 72.87 & 2.0160 & -23.4 \\
\hline OR & 68.00 & 0.5919 & -1.67 & 92.20 & 4.261 & -11.83 & 69.98 & -2.6850 & -21.6 \\
\hline SL-22 & 66.52 & -0.4955 & -7.61 & 92.07 & -3.204 & -5.23 & 78.81 & 4.9020 & 3.17 \\
\hline NS-101 & 71.16 & -0.2907 & 3.87 & 99.53 & 4.694 & -9.04 & 82.02 & -2.3530 & 36.6 \\
\hline MM & 75.14 & -0.905 & -6.56 & 102.20 & 0.198 & 0.62 & 63.94 & -4.1000 & -9.47 \\
\hline CAU-M-4 & 81.00 & -0.3995 & -6.52 & 106.80 & 3.007 & -6.44 & 60.12 & 1.9850 & -22.61 \\
\hline MS & 83.08 & 0.0535 & -8.6 & 112.73 & -5.491 & -10.6 & 62.96 & -0.0512 & -19.59 \\
\hline CAU-TS-2 & 82.53 & -0.065 & -8.23 & 115.20 & 2.286 & -11.58 & 68.85 & 1.4300 & -18.05 \\
\hline CAU-TS-5 & 83.93 & -0.0576 & -6.96 & 115.87 & -2.076 & -11.97 & 78.42 & 1.2970 & 25.71 \\
\hline CAU-TS-1-3 & 83.40 & 0.1799 & -7.69 & 117.60 & 0.088 & -11.74 & 90.97 & 3.1730 & -25.05 \\
\hline CAU-TS-2-3 & 83.40 & -0.1251 & -8.53 & 117.00 & 6.600 & -9.62 & 90.35 & -2.0660 & 6.14 \\
\hline CAU-TS-3-3 & 81.78 & 0.7914 & -4.49 & 112.93 & 4.271 & -12.73 & 75.73 & -2.8900 & 11.65 \\
\hline CAU-TS-4-3 & 74.48 & 0.862 & 2.63 & 111.07 & 1.380 & -5.39 & 66.01 & -1.4920 & -20.3 \\
\hline CAU-TS-4-3 & 72.07 & -0.1784 & -7.31 & 103.07 & 9.593 & -9.94 & 70.53 & 2.1280 & -8.04 \\
\hline CAU-TS-1 & 71.82 & 0.3788 & -9.06 & 96.47 & 0.331 & -11.52 & 74.87 & 0.0566 & -10 \\
\hline Mean+SE & & $75.66+1.34$ & & & $100.64+1.58$ & & $68+2.25$ \\
\hline
\end{tabular}

\begin{tabular}{|l|c|c|c|c|c|c|c|c|c|}
\hline & \multicolumn{3}{|c|}{$\begin{array}{c}\text { Number of primary branches } \\
\text { per plant }\end{array}$} & \multicolumn{3}{c|}{ Fruit length (cm) } & \multicolumn{3}{c|}{ Fruit diameter (cm) } \\
\hline \multicolumn{1}{|c|}{ Genotype } & Mean & bi & $\mathrm{S}^{2}$ di & Mean & bi & S $^{2}$ di & Mean & bi & S $^{2}$ di \\
\hline TWC-1 & 3.29 & 3.669 & -0.1 & 4.97 & -2.071 & 0.04 & 3.14 & 2.111 & -0.08 \\
\hline TWC-2 & 2.87 & 0.624 & -0.16 & 4.72 & 5.697 & -0.08 & 2.93 & -0.0302 & -0.06 \\
\hline TWC-3 & 3.02 & -0.615 & -0.09 & 3.25 & 5.919 & 0.25 & 2.55 & 2.017 & -0.11 \\
\hline TWC-4 & 3.07 & -1.180 & -0.12 & 2.70 & -2.620 & -0.14 & 2.50 & 1.469 & -0.04 \\
\hline TWC-8 & 3.11 & 0.692 & -0.17 & 3.64 & -5.026 & 0.03 & 3.46 & 3.930 & -0.11 \\
\hline TWC-9 & 2.67 & 1.742 & -0.01 & 4.27 & -0.302 & -0.15 & 3.83 & 1.450 & -0.08 \\
\hline TWC-10 & 3.31 & -5.936 & 0.71 & 4.13 & 2.622 & -0.09 & 3.24 & 1.775 & -0.1 \\
\hline TWC-11 & 4.13 & 2.827 & -0.12 & 3.40 & 2.453 & 0.13 & 3.86 & 0.0649 & -0.08 \\
\hline
\end{tabular}




\begin{tabular}{|c|c|c|c|c|c|c|c|c|c|}
\hline A.ALOK & 3.61 & -1.605 & -0.1 & 3.93 & -6.305 & 0.09 & 3.40 & 3.441 & -0.08 \\
\hline A.VIKAS & 3.32 & 2.706 & -0.11 & 4.44 & 1.828 & -0.11 & 3.99 & 0.054 & -0.08 \\
\hline BRH-1 & 2.87 & -0.056 & -0.16 & 4.11 & 1.810 & -0.14 & 3.67 & 1.249 & -0.03 \\
\hline BRH-2 & 3.13 & -2.699 & -0.11 & 3.42 & 3.509 & 0.1 & 2.82 & 3.509 & -0.08 \\
\hline PR & 3.80 & 1.111 & -0.19 & 3.53 & 5.825 & 0.39 & 2.67 & 4.920 & -0.08 \\
\hline SR & 3.38 & 1.861 & -0.07 & 3.16 & -3.621 & -0.14 & 2.63 & 4.054 & -0.05 \\
\hline OR & 3.56 & -2.404 & 0.06 & 3.96 & -2.446 & 0.05 & 3.65 & 2.213 & -0.09 \\
\hline NS-101 & 4.04 & 0.836 & -0.16 & 4.12 & -2.843 & 0.22 & 2.50 & 0.828 & -0.05 \\
\hline MM & 3.82 & 1.523 & -0.12 & 4.28 & 0.784 & 0.02 & 2.71 & 1.377 & -0.1 \\
\hline CAU-M-4 & 3.42 & 1.314 & -0.11 & 4.34 & -0.053 & -0.11 & 3.12 & 1.416 & 0 \\
\hline MS & 3.11 & -1.375 & -0.12 & 3.99 & 2.409 & -0.09 & 3.51 & 0.021 & 0.04 \\
\hline CAU-TS-2 & 3.49 & -0.211 & -0.03 & 3.75 & -0.586 & -0.14 & 2.80 & 2.741 & -0.08 \\
\hline CAU-TS-5 & 3.69 & -0.271 & -0.2 & 3.92 & -1.313 & -0.09 & 2.34 & 0.445 & -0.03 \\
\hline CAU-TS-4-3 & 3.42 & -0.758 & 0.02 & 4.21 & 3.085 & -0.11 & 2.82 & -0.044 & -0.06 \\
\hline CAU-TS-4-3 & 3.22 & -0.693 & 0.14 & 3.99 & -1.449 & -0.04 & 3.37 & 2.926 & -0.09 \\
\hline CAU-TS-1 & 3.29 & 1.589 & -0.08 & 4.21 & 0.0514 & -0.03 & 3.25 & 3.755 & -0.04 \\
\hline Mean+SE & \multicolumn{3}{|c|}{$3.72+0.20$} & \multicolumn{3}{|c|}{$3.99+0.17$} & \multicolumn{3}{|c|}{$3.37+0.15$} \\
\hline
\end{tabular}

\begin{tabular}{|l|c|c|c|c|c|c|c|c|c|}
\hline & Number fruits per plant & \multicolumn{3}{c|}{ Fruit weight (g) } & \multicolumn{3}{c|}{ Fruit yield per plant (g) } \\
\hline Genotype & Mean & bi & $\mathrm{S}^{2} \mathrm{di}$ & Mean & bi & $\mathrm{S}^{2} \mathrm{di}$ & Mean & bi \\
\hline TWC-1 & 11.38 & 5.376 & -1.54 & 39.36 & 2.997 & -27.05 & 220.60 & 6.210 & -1388.61 \\
\hline TWC-2 & 9.46 & -1.90 & -1.21 & 27.92 & 4.331 & 152.18 & 352.51 & 5.453 & 2760.79 \\
\hline TWC-3 & 13.42 & -6.782 & -0.85 & 18.14 & 0.543 & -24.65 & 426.65 & 0.254 & -2544.48 \\
\hline TWC-4 & 15.09 & 2.79 & -1.36 & 22.92 & 1.190 & -2.78 & 363.92 & -3.237 & 3668.74 \\
\hline TWC-8 & 14.31 & -1.30 & -1.63 & 30.28 & 1.302 & -21.7 & 333.19 & 1.878 & 1251.07 \\
\hline TWC-9 & 16.54 & -3.16 & -1.39 & 29.27 & 0.807 & -11.17 & 317.49 & 0.426 & -57.52 \\
\hline
\end{tabular}




\begin{tabular}{|c|c|c|c|c|c|c|c|c|c|}
\hline TWC-10 & 18.13 & -1.88 & -0.87 & 24.17 & 0.646 & -25.12 & 339.11 & 4.120 & -929.21 \\
\hline TWC-11 & 14.63 & 6.49 & -0.69 & 29.19 & 0.894 & 51.93 & 388.49 & 1.819 & -14.94 \\
\hline A.ALOK & 11.80 & -0.36 & -0.49 & 44.37 & 2.499 & 45.11 & 395.53 & 2.279 & -1019.86 \\
\hline A.VIKAS & 15.75 & -6.48 & -0.12 & 51.15 & 0.361 & -23.91 & 372.01 & -3.851 & -716.51 \\
\hline BRH-1 & 20.41 & -3.15 & -0.15 & 44.63 & 0.045 & 31.3 & 377.61 & 6.181 & -1510.23 \\
\hline BRH-2 & 20.98 & 1.77 & 0.36 & 31.82 & 1.995 & 23 & 457.34 & 1.546 & -721.96 \\
\hline PED & 20.55 & -1.32 & -0.97 & 30.87 & 1.350 & -22.27 & 530.03 & 1.802 & -885.38 \\
\hline PR & 21.33 & 0.16 & -1.63 & 29.39 & 0.622 & -16.7 & 517.65 & -4.272 & -2167.97 \\
\hline SR & 19.96 & 2.29 & -1.54 & 23.00 & 4.545 & -23 & 406.13 & -5.964 & 173.76 \\
\hline OR & 18.36 & 0.94 & -1.83 & 30.19 & 5.035 & 79.5 & 325.53 & 0.442 & -2595.73 \\
\hline SL-22 & 18.41 & -1.13 & -1.63 & 28.69 & 2.566 & 52.33 & 270.57 & -3.149 & -1570.51 \\
\hline NS-101 & 17.21 & 3.47 & -1.72 & 29.55 & 1.566 & 78.57 & 249.59 & 1.514 & -2897.93 \\
\hline MM & 17.18 & -2.91 & -1.19 & 45.71 & 4.309 & 9.84 & 300.03 & 1.016 & -615.11 \\
\hline CAU-M-4 & 19.66 & -1.93 & -1.75 & 39.08 & 4.239 & 102.1 & 283.19 & 3.016 & 1430.5 \\
\hline MS & 21.47 & -1.91 & -1.57 & 29.84 & 2.068 & -23.83 & 297.46 & 0.5464 & 352.73 \\
\hline CAU-TS-2 & 21.83 & 1.09 & -0.56 & 31.72 & 2.429 & -11.73 & 340.04 & 1.393 & -3002.79 \\
\hline CAU-TS-5 & 19.61 & 2.81 & -1.67 & 31.26 & 1.669 & -9.75 & 326.21 & 2.856 & -2961.4 \\
\hline CAU-TS-1-3 & 18.33 & 0.65 & -1.76 & 36.48 & 1.331 & 6.56 & 263.59 & -2.016 & -1247.7 \\
\hline CAU-TS-2-3 & 18.24 & -0.71 & -1.85 & 39.77 & 2.196 & -25.47 & 292.22 & 6.532 & -1472.61 \\
\hline CAU-TS-3-3 & 18.06 & 0.77 & -1.72 & 30.97 & 2.666 & 72.2 & 364.36 & 0.309 & -144.08 \\
\hline CAU-TS-4-3 & 18.62 & -1.38 & -0.51 & 21.28 & 1.371 & -26.54 & 276.73 & 7.454 & 921.1 \\
\hline CAU-TS-4-3 & 17.18 & 3.36 & -1.42 & 28.05 & 1.340 & 36.73 & 219.18 & 1.493 & -2741.32 \\
\hline CAU-TS-1 & 13.85 & 3.91 & -0.32 & 37.62 & 2.838 & -21.26 & 226.92 & 2.680 & -2551.95 \\
\hline Mean+SE & \multicolumn{3}{|c|}{$17.32+0.28$} & \multicolumn{3}{|c|}{$3.99+0.17$} & \multicolumn{3}{|c|}{$32.30+2.41$} \\
\hline
\end{tabular}


The stable genotypes for other important yield contributing characters were TWC-1, Arka Vikas and BRH-1 for fruit length; TWC-9 for fruit diameter and Arka Alok, CAU-TS-2-3 and CAU-TS-1 for fruit weight, respectively.

In the present study, highly stable performance for mean fruit yield per plant was shown by the genotypes Arka Vikas, TWC-8, TWC-11, BRH-2, Pusa Early Dwarf and CAU-TS-2 with mean yield more than the population mean with unit regression coefficient and non-significant deviation from the regression. Good performing variety Pusa Ruby came out to be quite unstable as the regression coefficient was significantly deviated from unity though deviation from regression was non-significant implying that this variety could be recommended for poor environment. Likewise, Sutton Roma could also be recommended for poor environment. Similar observations were observed by Shalini (2016), Shankar et al., (2017) and Sonam et al., (2017).

From the above discussion, it could be concluded that no single genotype was stable for all the characters under study. This is in consonance with studies of Ummyiah et.al. (2015), Ravindra Kumar et al., (2019) and Krupal and Acharya (2019) as they observed that no single genotype showed stability for all the traits under different environments.

It can be concluded that variety BRH-2, which was stable for the characters fruit yield per plant and number of fruits per plant and Arka Vikas which was stable for fruit length and fruit yield per plant can be utilized to develop stable strains having wider adaptability in future breeding programmes. It can also be concluded that November sowing showed better performance in yield while September sowing favoured for earliness, better growth, and can be suitable for growing genotypes for seed production purpose.

\section{References}

Allard, R.W. and A.D. Bradshaw. 1964. Implications of genotype-environmental interactions in applied plant breeding. Crop Sci. 4:503-508

Eberhart, S.A. and W.A. Russell. 1966. Stability parameters for comparing varieties. Crop Sci. 6:36-40

Finlay, K.W. and G.N. Wilkinson.1963. The analysis of adaptation in a plant breeding programme. Aust J Agric Res. 14:742754.

Krupal C Bhalala and R.R. Acharya.2019. Stability analysis in tomato (Solanum lycopersicum L.). Journal of Pharmacognosy and Phytochemistry 8(3): 4776-4784.

Shalini M.2016. Stability analysis in tomato (Solanum lycopersicum L.) for yield and yield attributing traits. Environment and Ecology 34(4B):2037-2043

Shankar, A., R.V.S.K., Reddy,P. Saidaiah, K. Uma Krishna and K. Uma Jyothi. 2017. Study on Stability for Yield and Its Components in Tomato (Solanum lycopersicum L.). Int.J.Curr.Microbiol.App.Sci. 6(9): 804812

Sonam Spaldon, R.K. Samnotra, Rinchan Dolkar and Deshraj Choudhary.2017. Stability Analysis and Genotype X Environment Interaction of Quality Traits in Tomato (Solanum lycopersicum L.). Int.J.Curr.Microbiol.App.Sci. 6(2): 15061515.

Ravindra Kumar, S.K. Singh and K. Srivastava. 2019. Stability Analysis in Tomato Inbreds and Their $F_{1} S$ for Yield and Quality Traits. Agricultural Research 2019; 8:141-147.

Ummyiah, H.M., Nayeema Jabeen, Kousar Parveen and S.H. Khan.2015. Stability analysis and genotype $\mathrm{x}$ environment interaction of some tomato varieties under Kashmir conditions. Green Farming 6 (5): 931-934. 


\section{How to cite this article:}

Brajendra Singh, N., Joyashree Dey, Herojit Singh Athokpam and Laishram, J. M. 2021. Stability Analysis for Fruit Yield and its Components in Tomato (Solanum lycopersicum L.) under Acidic Soils of Manipur Valley. Int.J.Curr.Microbiol.App.Sci. 10(01): 2243-2250. doi: https://doi.org/10.20546/ijcmas.2021.1001.258 